\title{
Unitary evolution of free massless fields in de Sitter space-time
}

\author{
Daniel Gómez Verge** \\ Instituto de Estructura de la Materia, \\ CSIC, Serrano 121, 28006 Madrid, Spain \\ Eduardo J. S. Villaseñon \\ Grupo de Modelización y Simulación Numérica, Universidad Carlos III de Madrid, \\ Avda. de la Universidad 30, 28911 Leganés, Spain and \\ Instituto de Estructura de la Materia, \\ CSIC, Serrano 123, 28006 Madrid, Spain
}

(Dated: February 26, 2008)

\begin{abstract}
We consider the quantum dynamics of a minimally coupled massless scalar field in de Sitter space-time. The classical evolution is represented by a canonical transformation on the phase space for the field theory. By studying the corresponding Bogoliubov transformations, we show that the symplectic map that encodes the evolution between two instants of time cannot be unitarily implemented on any Fock space built from a $S O(4)$-symmetric complex structure. We will show also that, in contrast with some effectively lower dimensional examples arising from Quantum General Relativity such as Gowdy models, it is impossible to find a time dependent conformal redefinition of the massless scalar field leading to a quantum unitary dynamics.

PACS numbers: 04.62.+v, 04.60.Ds, 98.80.Qc
\end{abstract}

\section{INTRODUCTION}

One of the most surprising features of any Fock quantization of a linear symplectic dynamical system with infinitely many degrees of freedom is the impossibility of defining the unitary quantum counterpart of every linear symplectic transformation [1]. In the special case of a field theory on a four dimensional space-time background, this characteristic behavior of the infinite dimensional systems is responsible for the generic impossibility to make sense of the unitary quantum evolution operator from an initial Cauchy surface to any final Cauchy surface, both for curved and flat background space-times [2]. This is the case for scalar fields propagating in Minkowski space-time if the Cauchy surfaces are not level surfaces of some Minkowskian time [3].

The problem of the unitary implementability of symplectic transformations lies also at the heart of many recent interesting results concerning the quantization of some midi-superspace

* dgvergel@iem.cfmac.csic.es

†ejsanche@math.uc3m.es 
models [4, 5, 6, 7, 8, 9, 10, 11, 12, 13, 14, 15]. Among these symmetry reductions of general relativity, linearly polarized Gowdy models [16, 17] have been the focus of intensive study due to their cosmological interpretation and exact solvability. After partial gauge fixing and deparameterization ${ }^{1}$, the field-like degrees of freedom of the Gowdy models admit a natural interpretation as a massless scalar field $\phi$ propagating in a globally hyperbolic $(1+2)$-background $\left(I_{\Sigma} \times \Sigma, g_{a b}^{\Sigma}\right)$. The scalar field dynamics has to satisfy an additional symmetry condition with respect to a certain Killing vector field of the background metric. The topology of the spatial Cauchy surfaces $\{t\} \times \Sigma$ is constrained to be homeomorphic to $\mathbb{T}^{2}=\mathbb{S}^{1} \times \mathbb{S}^{1}$ (for the so-called Gowdy $\mathbb{T}^{3}$ model) or $\mathbb{S}^{2}$ (for the Gowdy $\mathbb{S}^{1} \times \mathbb{S}^{2}$ and $\mathbb{S}^{3}$ models). The time interval $I_{\Sigma} \subset \mathbb{R}$, parameterized by a global coordinate $t$, is respectively $I_{\mathbb{T}^{2}}=(0, \infty)$ or $I_{\mathbb{S}^{2}}=(0, \pi)$. Finally, the space-time metric $g_{a b}^{\Sigma}$ has a very simple form

$$
g_{a b}^{\Sigma}=f_{\Sigma}^{-4}(t) \stackrel{\circ}{g}_{a b}^{\Sigma}=f_{\Sigma}^{-4}(t)\left(-(\mathrm{d} t)_{a}(\mathrm{~d} t)_{b}+\gamma_{a b}^{\Sigma}\right),
$$

where $\gamma_{a b}^{\Sigma}$ is the standard flat metric when $\Sigma$ is the 2-torus, and the round metric when $\Sigma$ is the 2 -sphere. The fuction $f_{\Sigma}$ is a time dependent conformal factor whose explicit value is $f_{\mathbb{T}^{2}}(t)=1 / \sqrt{t}$ or $f_{\mathbb{S}^{2}}(t)=1 / \sqrt{\sin t}$, depending on the spatial topology. At this point, it is worthwhile noting that the background metric of the $\Sigma=\mathbb{S}^{2}$ models is somehow similar to the $(1+3)$-dimensional de Sitter metric in the sense that the metric (1.1) is conformally equivalent to the Einstein static $(1+2)$-universe. In all these cases, it is impossible to find a Fock quantization using a complex structure compatible with the spatial symmetries of the Riemannian metrics $\gamma_{a b}^{\Sigma}$, such that the quantum evolution (in terms of the time coordinate t) of the massless field $\phi$ is unitarily implementable ${ }^{2}$. However, irrespective of $\Sigma$, it is always possible to avoid this problem by performing a suitable time-dependent field redefinition that preserves the spatial homogeneity and isotropy ${ }^{3}$. This is given by $\phi(t, s)=f_{\Sigma}(t) \cdot \zeta(t, s)$ -here $(t, s) \in I_{\Sigma} \times \Sigma$. When this is done, there exists a unique (modulo unitary equivalence) Fock quantization for the new field $\zeta$ such that the quantum time evolution can be unitarily implemented [11, 15].

Although the previous results were obtained in the restricted context of the quantization of Gowdy models, one could expect that the techniques described above have a wider range of applicability. In particular, they could be used to identify -and possibly solve- the problems associated with the failure of the unitary implementation of time evolution in the Fock quantizations on sufficiently symmetric curved backgrounds. In this respect, the most ambitious program would be to formulate a theorem classifying the class of space-times where a conformal redefinition of the fields solves the problem of unitarity of evolution. However, before starting such a general program, it is convenient to analyze concrete models to acquire some familiarity with the different possible behaviors and pathologies. In particular, it is important to clarify if the dimensionality of the space-time plays a key role in this process

${ }^{1}$ The deparameterization program was done for the first time in the context of the $\mathbb{T}^{3}$ Gowdy model 6, 8, 10], and generalized later for the $\mathbb{S}^{1} \times \mathbb{S}^{2}$ and $\mathbb{S}^{3}$ cases [14].

2 This was first shown for the $\mathbb{T}^{3}$ model [7, 12] and later on seen to be true also in the $\mathbb{S}^{1} \times \mathbb{S}^{2}$ and $\mathbb{S}^{3}$ Gowdy topologies [15].

${ }^{3}$ Again, these field redefinitions were firstly used to deal with the problem of the unitary implementability of the quantum dynamics for the $\mathbb{T}^{3}$ Gowdy model [9] and later on generalized -and interpreted on geometric grounds as conformal transformations- for the remaining topologies [15]. 
since, up to now, only $(1+2)$-dimensional cases have been analyzed. The main aim of this paper is precisely to test the techniques developed in the context of Gowdy models in a $(1+3)$-dimensional example. To this end, we will consider the class of Fock quantizations for the minimally coupled massless scalar field propagating in the de Sitter space-time, not only owing to its similarity to Gowdy models, but also due to its intrinsic interest.

The terms 'mass' and 'massless' deserve special attention when we are dealing with a curved background. This is so because the physical quantity called mass can be associated both to the Galilean or Poincare groups but it does not exist, by itself, as a conserved quantity in a generic space-time. Nonetheless, it is possible to make sense of the mass in de Sitter space by following an ambiguity free limit process in which the space-time becomes flat [18]. In this context, the scalar field that we are going to consider in this paper is not really a massless field in the above sense -in particular, its field equation is not conformally invariant- but corresponds to the unitary irreducible representation associated with the zero-value of the Casimir operator (that is proportional to the Laplace-Bertrami operator) of the de Sitter group [19]. Although this scalar field is not massless, it arises in a natural form in the context of the massless spin-two fields in the de Sitter space and it is usually called the minimally coupled massless scalar field.

The problem of quantizing this system has been profusely analyzed in the literature [19, $20,21,22,23,24,25,26]$. The scalar quantum field $\hat{\phi}(x)$, understood as an operator-valued distribution on certain Hilbert space $\mathcal{H}$, must be a solution to the classical field equations (that we will discuss in section III) and satisfy certain reasonable physical conditions that can be summarized as follows.

c1) Microcausality. The field commutator $\left[\hat{\phi}\left(x_{1}\right), \hat{\phi}\left(x_{2}\right)\right]$ must vanish if the points $x_{1}$ and $x_{2}$ of the de Sitter space-time are not causally connected.

c2) Covariance. There exists a unitary representation $\hat{D}(g)$ of the de Sitter group $O(1,4)$ such that the field is covariant, i.e. $\hat{D}(g) \hat{\phi}(x) \hat{D}^{-1}(g)=\hat{\phi}(g \cdot x)$ for all $g \in O(1,4)$, where we have denoted by $g \cdot x$ the action of $g$ on the point $x$ of the de Sitter space.

c3) Invariance of the vacuum. There exists a cyclic unit vector $|0\rangle \in \mathcal{H}$ which is invariant under the representation $\hat{D}$, i.e. $\hat{D}(g)|0\rangle=|0\rangle, \forall g \in O(1,4)$.

c4) Hadamard condition. The Hadamard restriction demands that the two-point functions $\left\langle 0\left|\hat{\phi}\left(x_{1}\right) \hat{\phi}\left(x_{2}\right)\right| 0\right\rangle$ have the right short-distance singularity behavior as $x_{1} \rightarrow x_{2}$. This condition allows us to define the expectation value of the stress-energy operator in a completely satisfactory manner.

It can be shown that, once a non-divergent, complete set of solutions to the equation of motion has been fixed, it is impossible to construct a de Sitter-invariant Fock representation for which the vacuum state is of Hadamard type [22, 23]. The origin of the problem is the existence of zero-modes, which is related to the fact that the wave equation has constant solutions. Therefore, a fully covariant quantization requires a type of representations of the canonical commutation relations different from those used in any Fock-like approach [19, 26]. The Gupta-Bleuer-type quantization scheme, rigourously formulated in [19], successfully incorporated the conditions c1)-c4) stated above in a non-Fock representation. However, even if it is possible to obtain a fully covariant quantization through non-Fock representations, 
we believe that it is necessary and interesting to probe if Fock quantizations based in some suitable weakened version of covariance can provide a well defined and unitary quantum dynamics in this system. For instance, following [23], if one adopts a weaker version of covariance by restricting the condition c2) only to the subgroup $O(4)$ it is not necessary to abandon the usual Fock quantization. Of course, according to [2], in those cases if the Fock vacua are assumed to be of Hadamard type, it is expected that the symplectic transformation defined by the time evolution cannot be, in general, unitarily implemented. Hence, if we want to have a unitary evolution, it is important to avoid restricting the discussion to the Hadamard case.

The differences of our treatment with respect to previous works available in literature can be summarized as follows. We have not restricted ourselves to the de Sitter-invariant case but we have considered all the Fock quantizations for which the one-particle Hilbert space is obtained from a $S O(4)$-invariant complex structure. Furthermore, we do not require the states to be of Hadamard type (a physically well justified hypothesis that nevertheless makes the unitary implementability of dynamics difficult [2]). In addition, in order to study the unitary implementability of the quantum evolution, we explore all the possible homogeneous reparameterizations of the minimally coupled scalar field.

Before going further, it is important to discuss to what extent the treatment for the Gowdy models introduced above can be followed in the de Sitter case. For the vacuum Gowdy models the background metric is fiducial (i.e. it is a by-product of the deparameterization process) and the massless scalar field has only a geometric interpretation, but not a physical one. Therefore, we are free to perform conformal redefinitions of the form

$$
g_{a b}^{\Sigma} \mapsto \stackrel{\circ}{g}_{a b}^{\Sigma}=f_{\Sigma}^{4} g_{a b}^{\Sigma}, \quad \phi \mapsto \zeta=f_{\Sigma}^{-1} \phi
$$

that modify both the background metric and the scalar field. However, in the context of quantum field theory in curved space-times, both the matter fields and the background metric have a clear physical interpretation and are chosen to describe a concrete physical situation. Hence, a priori, the class of conformal transformations considered in order to deal with the Gowdy models are in this case more difficult to justify. The only acceptable reason for modifying the background and the matter field would be to avoid some problems associated with the original theory, such as the failure of the unitary implementation of the quantum dynamics.

The paper is organized in the following way. After this introduction, we will review in section II the properties of the de Sitter space-time that are used in the body of the paper. We will discuss in section III the different formulations describing a minimally coupled massless scalar field propagating in de Sitter space-time that are available after performing a conformal re-scaling of the massless field. In section IV we will explicitly characterize all the Fock quantizations of the massless scalar field that respect the $S O(4)$ symmetry of the spatial slices of the de Sitter background. The main result of the paper is presented in section $\nabla$ where we will show that, irrespective of the conformal factor of the massless scalar, it is impossible to have a quantum unitary evolution operator. Notice that this result affects the two-parameter family of $O(4)$-invariant Hadamard representations characterized in [23]. We will end the paper with our conclusions in section VI. 


\section{DE SITTER SPACE-TIME}

Space-time metrics $g_{a b}$ of constant curvature are locally characterized by the vanishing of their Weyl curvature. In $(1+3)$ dimensions this implies

$$
R_{a b}-\frac{1}{4} R g_{a b}=0, \quad R \text { constant. }
$$

The solution to (2.1) that corresponds to positive constant curvature $R>0$ is the so-called de Sitter space-time $\mathrm{dS}(R)$. This one-parameter family of space-times can be explicitly constructed as the warped product $\mathbb{R} \times_{\kappa_{R}} \mathbb{S}^{3}$ of the real line, with metric element $-\mathrm{d} t^{2}$, and the round 3 -sphere by considering the warping functions ${ }^{4}$

$$
\kappa_{R}(t)=\frac{12}{R} \cosh ^{2}\left(\sqrt{\frac{R}{12}} t\right) .
$$

In other words

$$
g_{a b}=-(\mathrm{d} t)_{a}(\mathrm{~d} t)_{b}+H^{-2} \cosh ^{2}(H t) \gamma_{a b},
$$

where $H:=\sqrt{R / 12}$ is the Hubble constant and $\gamma_{a b}$ is the pullback to $\mathrm{dS}(R)$ of the round metric of the unit 3-sphere by means of the canonical projection $\mathbb{R} \times \mathbb{S}^{3} \rightarrow \mathbb{S}^{3}$. The spatial slices of constant time coordinate $t$ foliate $\mathrm{dS}(R)$ by a one-parameter family of Cauchy surfaces all of them diffeomorphic to $\mathbb{S}^{3}$. Their geodesic normals contract monotonically to a minimum spatial separation at $t=0$, and then re-expand exponentially to infinity. In view of (2.1), $\mathrm{dS}(R)$ can be regarded as a solution of vacuum Einstein's equation with a positive cosmological constant $\Lambda=R / 4>0$ which sets the expansion rate to $H \propto \sqrt{\Lambda}$. In this context, if the observed acceleration of our universe can be explained in terms of a cosmological constant, its evolution may be described by a de Sitter solution at the infinite future.

The structure of the conformal infinity of $\mathrm{dS}(R)$ can be easily obtained from (2.2) by redefining the global time coordinate $t \in \mathbb{R}$ in terms of a new global time coordinate $T \in$ $(-\pi / 2, \pi / 2)$ through

$$
T=2 \arctan (\tanh (H t / 2)), \quad-\pi / 2<T<\pi / 2 .
$$

In terms of this coordinate it is clear that

$$
g_{a b}=H^{-2} \cosh ^{2}(H t) \bar{g}_{a b}, \quad \text { where } \bar{g}_{a b}:=-(\mathrm{d} T)_{a}(\mathrm{~d} T)_{b}+\gamma_{a b} .
$$

Therefore, denoting by $E:=\left(\mathbb{R} \times \mathbb{S}^{3}, \bar{g}_{a b}\right)$ the Einstein static universe, with $-\infty<T<\infty$, the de Sitter space is conformal to the submanifold $\left((-\pi / 2, \pi / 2) \times \mathbb{S}^{3}, \bar{g}_{a b}\right) \subset E$. Then, the surfaces $T= \pm \pi / 2$ act as future and past space-like infinities for time-like and null lines in de Sitter space-time. Due to this fact, and in contrast to Minkowski space, both particle and event horizons exist in the de Sitter space-time for geodesic families of observers [27].

In the following we will adimensionalize the time coordinate by changing $t \mapsto H t$, and

\footnotetext{
${ }^{4}$ We will choose units such that $c=\hbar=1$.
} 
choose units such that $H=1$. Then we can write

$$
g_{a b}=-(\mathrm{d} t)_{a}(\mathrm{~d} t)_{b}+\cosh ^{2} t \gamma_{a b} .
$$

\section{SCALAR FIELD ON DE SITTER BACKGROUND}

In this paper we will focus on the dynamics of free minimally coupled massless scalar fields propagating in a de Sitter background. To this end, let us consider the standard classical action

$$
S(\phi)=-\frac{1}{2} \int_{\left[t_{0}, t_{1}\right] \times \mathbb{S}^{3}}|g|^{1 / 2} g^{a b}(\mathrm{~d} \phi)_{a}(\mathrm{~d} \phi)_{b}
$$

for a smooth free massless scalar field $\phi \in C^{\infty}\left(\mathbb{R} \times \mathbb{S}^{3} ; \mathbb{R}\right)$ evolving from $t_{0}$ to $t_{1}$ in a de Sitter space-time $\left(\mathbb{R} \times \mathbb{S}^{3}, g_{a b}\right)$, where $g_{a b}$ is given by $(\underline{2.5})$. The massless field $\phi$ satisfies then the linear wave equation

$$
g^{a b} \nabla_{a} \nabla_{b} \phi=0
$$

where $\nabla_{a}$ is the Levi-Civita connection associated to $g_{a b}$. It is well known [28] that equation (3.2) has a well possed initial value formulation in terms of the Cauchy data defined on some level surface of the coordinate field $t$. In the following subsections we will discuss certain alternative descriptions of this system and analyze in detail the structure of its covariant and canonical phase space formulations.

\section{A. Conformal field redefinitions}

As we have mentioned in the introduction, one of the main lessons that we have learned from the study of the quantization of the Gowdy models is that, in order to have a well defined unitary quantum dynamics, it is imperative to consider a new auxiliary field $\zeta$ related to the original one in terms of a conformal transformation [14]. In the case of a massless scalar field propagating in the de Sitter space-time, we will restrict ourselves to spatially homogeneous field redefinitions of the form

$$
\phi(t, s)=f(t) \zeta(t, s), \quad(t, s) \in \mathbb{R} \times \mathbb{S}^{3}
$$

with $f(t)>0$ a (fixed) smooth, positive definite, real-valued function that we will try to fix by demanding that the quantum theory be well behaved.

The classical field dynamics for $\zeta$ can be derived from the variational principle

$$
\begin{aligned}
s(\zeta) & :=S(\phi)=S(f \zeta) \\
& =-\frac{1}{2} \int_{\left[t_{0}, t_{1}\right] \times \mathbb{S}^{3}}|\stackrel{\circ}{g}|^{1 / 2} \stackrel{\circ}{g} a b\left((\mathrm{~d} \zeta)_{a}(\mathrm{~d} \zeta)_{b}+2(\mathrm{~d} \log f)_{a} \zeta(\mathrm{d} \zeta)_{b}+(\mathrm{d} \log f)_{a}(\mathrm{~d} \log f)_{b} \zeta^{2}\right)
\end{aligned}
$$

that can be easily obtained by plugging (3.3) in (3.1). Here $\stackrel{\circ}{g}_{a b}:=f^{2} g_{a b}$ is a space-time metric on $\mathbb{R} \times \mathbb{S}^{3}$ conformal to the de Sitter one. Notice that the field $\zeta$ can be interpreted as a scalar field propagating on the metric background defined by $\stackrel{\circ}{g}_{a b}$ and coupled, in a non-standard way, to the time-dependent potential $\log f$. The massless field equation (3.2) 
for $\phi$ can be equivalently rewritten in terms of $\zeta$ in the form

$$
\stackrel{\circ}{g}^{a b} \stackrel{\circ}{\nabla}_{a} \stackrel{\circ}{\nabla}_{b} \zeta+\left(\stackrel{\circ}{g}^{a b} \stackrel{\circ}{\nabla}_{a} \stackrel{\circ}{\nabla}_{b} f-\stackrel{\circ}{g}^{a b}(\mathrm{~d} \log f)_{a}(\mathrm{~d} \log f)_{b}\right) \zeta=0,
$$

where $\stackrel{\circ}{\nabla}_{a}$ is the Levi-Civita connection associated to $\stackrel{\circ}{g}_{a b}=f^{2} g_{a b}$. Equation (3.5) admites the interpretation of a Klein-Gordon like equation for the field $\zeta$-in the background $\stackrel{\circ}{g}_{a b^{-}}$ with a (generically) nonconstant 'mass term'. There are at least two special cases that deserve special attention. First, if we take $f(t)=1$ we recover the original massless scalar

field propagating in de Sitter space-time. On the other hand, in view of (2.4), if we choose $f(t)=1 / \cosh t$ the metric $\stackrel{\circ}{g}_{a b}$ becomes the metric of the Einstein static universe.

The action (3.4) can be equivalently written as

$$
\begin{aligned}
s(\zeta)=\frac{1}{2} \int_{t_{0}}^{t_{1}} \mathrm{~d} t \int_{\mathbb{S}^{3}}|\gamma|^{1 / 2}\left(f^{2} \cosh ^{3}(t) \dot{\zeta}^{2}+2 f \dot{f} \cosh ^{3}(t) \dot{\zeta \zeta}\right. \\
\left.\quad+f^{2} \cosh (t) \zeta \Delta_{\mathbb{S}^{3}} \zeta+\dot{f}^{2} \cosh ^{3}(t) \zeta^{2}\right),
\end{aligned}
$$

where the dot denotes the Lie derivative along $(\partial / \partial t)^{a}$ and $\Delta_{\mathbb{S}^{3}}$ is the Laplace-Beltrami operator on the round unit 3-sphere.

\section{B. Covariant phase space}

The space $\mathcal{S}$ of smooth real solutions to the equation of motion derived from the action (3.6) has the structure of an infinite-dimensional real vector space. Any $\zeta \in \mathcal{S}$ is a real smooth function on $\mathbb{R} \times \mathbb{S}^{3}$ that satisfies the wave equation

$$
\ddot{\zeta}+\left(3 \tanh (t)+2 \frac{\dot{f}(t)}{f(t)}\right) \dot{\zeta}+\left(3 \tanh (t) \frac{\dot{f}(t)}{f(t)}+\frac{\ddot{f}(t)}{f(t)}-\frac{\Delta_{\mathbb{S}^{3}}}{\cosh ^{2}(t)}\right) \zeta=0 .
$$

The variational principle (3.6) gives rise also to a natural (weakly) symplectic structure $\Omega$ on $\mathcal{S}$ defined by

$$
\Omega\left(\zeta_{1}, \zeta_{2}\right):=f^{2}(t) \cosh ^{3}(t) \int_{\mathbb{S}^{3}}|\gamma|^{1 / 2} \imath_{t}^{*}\left(\zeta_{1} \dot{\zeta}_{2}-\zeta_{2} \dot{\zeta}_{1}\right),
$$

where $\imath_{t}$ denotes the standard inclusion $\imath_{t}: \mathbb{S}^{3} \rightarrow \mathbb{R} \times \mathbb{S}^{3}, \imath_{t}(s)=(t, s)$. It is easy to show that $\Omega$ does not depend upon the choice of time $t$ used to define the embedding $\imath_{t}\left(\mathbb{S}^{3}\right) \subset \mathbb{R} \times \mathbb{S}^{3}$. The (infinite dimensional) linear symplectic space $\Gamma=(\mathcal{S}, \Omega)$ is called the covariant phase space of the system.

\section{Canonical phase space}

Let $\Upsilon=(\mathcal{P}, \omega)$ be the canonical phase space of smooth initial Cauchy data on the 3-sphere

$$
\mathcal{P}:=\left\{(Q, P) \mid Q, P \in C^{\infty}\left(\mathbb{S}^{3} ; \mathbb{R}\right)\right\},
$$


endowed with the standard (weakly) symplectic structure

$$
\omega\left(\left(Q_{1}, P_{1}\right),\left(Q_{2}, P_{2}\right)\right):=\int_{\mathbb{S}^{3}}|\gamma|^{1 / 2}\left(Q_{1} P_{2}-Q_{2} P_{1}\right) .
$$

Once a time $t$ has been specified, it is possible to construct a symplectomorphism between the spaces $\Gamma$ and $\Upsilon$. The isomorphism $\mathfrak{J}_{t}: \Gamma \rightarrow \Upsilon$, that takes a solution $\zeta \in \Gamma$ and finds the Cauchy data induced on $\mathbb{S}^{3}$ by virtue of the embedding $\imath_{t}: \mathbb{S}^{3} \rightarrow \mathbb{R} \times \mathbb{S}^{3}$,

$$
\begin{aligned}
& \mathfrak{J}_{t}: \Gamma \rightarrow \Upsilon, \quad \zeta \mapsto(Q, P)=\mathfrak{J}_{t}(\zeta), \\
& \text { where } \quad Q=\imath_{t}^{*} \zeta, \quad P=f^{2}(t) \cosh ^{3}(t) \imath_{t}^{*} \dot{\zeta}+f(t) \dot{f}(t) \cosh ^{3}(t) \imath_{t}^{*} \zeta,
\end{aligned}
$$

is (irrespective of the value of $t$ ) a symplectic transformation from $\Gamma$ to $\Upsilon$, i.e. $\Omega=\mathfrak{J}_{t}^{*} \omega$. The description of the classical dynamics in $\Upsilon$ is done in the form a non-autonomous Hamiltonian system $(\mathbb{R} \times \Upsilon, \omega, H(t))$, with time-dependent Hamiltonian $H(t): \Upsilon \rightarrow \mathbb{R}$ given by the quadratic form

$$
H(Q, P ; t)=\frac{1}{2} \int_{\mathbb{S}^{3}}|\gamma|^{1 / 2}\left(\frac{P^{2}}{f^{2}(t) \cosh ^{3}(t)}-2 \frac{\dot{f}(t)}{f(t)} P Q-f^{2}(t) \cosh (t) Q \Delta_{\mathbb{S}^{3}} Q\right)
$$

Notice that when $\dot{f} \neq 0$ the Hamiltonian (3.11) is indefinite.

It is important to point out that it is only a matter of convenience whether we describe dynamics using the covariant or the canonical phase space. For the most part of this paper, we will study the dynamical aspects of the system by using the covariant phase space. In particular, we will discuss in some detail in section $\mathrm{V}$ the classical evolution and its quantum counterpart.

\section{Mode decomposition}

Any vector $\zeta \in \Gamma$ is a smooth function on $\mathbb{R} \times \mathbb{S}^{3}$. Then, for each fixed value of $t \in \mathbb{R}$, $\imath_{t}^{*} \zeta \in C^{\infty}\left(\mathbb{S}^{3} ; \mathbb{R}\right)$. It is well known that any smooth function on $\mathbb{S}^{3}$ can be written in the form

$$
\imath_{t}^{*} \zeta(s)=\zeta(t, s)=\sum_{k=0}^{\infty} \sum_{l=0}^{k} \sum_{m=-l}^{l} A_{k l m}(t) Y_{k l m}(s) .
$$

Here $Y_{k l m}$ denote the standard spherical harmonics on $\mathbb{S}^{3}[29]$. They are eigenvectors of the Laplacian, $\Delta_{\mathbb{S}^{3}} Y_{k l m}=-k(k+2) Y_{k l m}$, and verify the $L^{2}\left(\mathbb{S}^{3}\right)$-orthogonality condition $(\delta$ denotes the Kronecker delta)

$$
\int_{\mathbb{S}^{3}}|\gamma|^{1 / 2} \bar{Y}_{k_{1} l_{1} m_{1}} Y_{k_{2} l_{2} m_{2}}=\delta\left(k_{1}, k_{2}\right) \delta\left(l_{1}, l_{2}\right) \delta\left(m_{1}, m_{2}\right) .
$$


They also satisfy ${ }^{5} \bar{Y}_{k l m}=Y_{k l-m}$. The complex coefficients $A_{k l m}(t)$ appearing in (3.12) are defined in terms of $\zeta$ through

$$
A_{k l m}(t)=\int_{\mathbb{S}^{3}}|\gamma|^{1 / 2} \bar{Y}_{k l m} \imath_{t}^{*} \zeta
$$

From (3.14) it is clear that $A_{k l m}(t)$ are smooth functions on $t \in \mathbb{R}$ that satisfy

$$
\begin{aligned}
& \bar{A}_{k l m}(t)=A_{k l-m}(t), \\
& \lim _{k \rightarrow \infty} \frac{1}{k^{p}} \frac{\mathrm{d}^{n} A_{k l m}}{\mathrm{~d} t^{n}}(t)=0, \quad \forall p, n \in \mathbb{N}_{0}, \quad \forall t \in \mathbb{R} .
\end{aligned}
$$

The condition (3.15) comes from the reality of the field $\zeta$. On the other hand, condition (3.16) is derived from the fact that, for any fixed value of $t, \partial_{t}^{n} \zeta(t, \cdot)$ is a smooth function on $\mathbb{S}^{3}$. Then, it is clear that any $\zeta \in \Gamma$ can be expanded in the form

$$
\zeta(t, s)=\sum_{k=0}^{\infty} \sum_{l=0}^{k} \sum_{m=-l}^{l}\left(a_{k l m} y_{k}(t) Y_{k l m}(s)+\overline{a_{k l m} y_{k}(t) Y_{k l m}(s)}\right), \quad(t, s) \in \mathbb{R} \times \mathbb{S}^{3},
$$

where the complex smooth functions $y_{k}$ are solutions to the equation

$$
\ddot{y}_{k}+\left(3 \tanh (t)+2 \frac{\dot{f}(t)}{f(t)}\right) \dot{y}_{k}+\left(3 \tanh (t) \frac{\dot{f}(t)}{f(t)}+\frac{\ddot{f}(t)}{f(t)}+\frac{k(k+2)}{\cosh ^{2}(t)}\right) y_{k}=0,
$$

whose real and imaginary parts are taken to be linearly independent, and the complex coefficients $a_{k l m}$ satisfy ${ }^{6}$

$$
\lim _{k \rightarrow \infty} \frac{1}{k^{p}}\left(a_{k l m} \frac{\mathrm{d}^{n} y_{k}}{\mathrm{~d} t^{n}}(t)+\bar{a}_{k l-m} \frac{\mathrm{d}^{n} \bar{y}_{k}}{\mathrm{~d} t^{n}}(t)\right)=0, \quad \forall p, n \in \mathbb{N}_{0}, \quad \forall t \in \mathbb{R} .
$$

By explicitly decomposing $y_{k}=u_{k}+i v_{k}$, with $u_{k}$ and $v_{k}$ real, the Wronskian of $u_{k}$ and $v_{k}$ can be written in terms of the Wronskian of $y_{k}$ and $\bar{y}_{k}$

$$
2 i W_{k}\left(t ; u_{k}, v_{k}\right):=2 i\left(\dot{v}_{k}(t) u_{k}(t)-\dot{u}_{k}(t) v_{k}(t)\right)=\bar{y}_{k}(t) \dot{y}_{k}(t)-\dot{\bar{y}}_{k}(t) y_{k}(t) .
$$

By virtue of (3.18), $W_{k}$ verifies the differential equation

$$
\dot{W}_{k}+\left(3 \tanh (t)+2 \frac{\dot{f}(t)}{f(t)}\right) W_{k}=0
$$

5 The bar denotes complex conjugation.

${ }^{6}$ Form the point of view of the classical theory, these conditions are needed to guarantee the smoothness of the solutions to the field equations. However, we do not need to know them in detail to discuss the quantized the model. In fact they will be relaxed to the milder condition $\sum_{k l m}\left|a_{k l m}\right|^{2}<\infty$ when we introduce the one-particle Hilbert space. 
It is then possible to write

$$
W_{k}\left(t ; u_{k}, v_{k}\right)=f^{-2}(t) \cosh ^{-3}(t) W_{k}^{0}
$$

with $W_{k}^{0} \in \mathbb{R}$. We will normalize the pairs $\left(u_{k}, v_{k}\right)$ by imposing

$$
W_{k}\left(t ; u_{k}, v_{k}\right)=\frac{1}{2} f^{-2}(t) \cosh ^{-3}(t), \quad \forall k \in \mathbb{N}_{0}
$$

It could appear that this condition is rather arbitrary but, as we will see, it is convenient to make this choice to ensure that the set of complex solutions

$$
\left\{\phi_{k l m}=y_{k} Y_{k l m} \mid k \in \mathbb{N}_{0}, l \in\{0, \ldots, k\}, m \in\{-l, \ldots, l\}\right\}
$$

is an orthonormal basis of the one-particle Hilbert space used to construct the physical Fock space of the system. In concrete, a possible election satisfying (3.20) is given by

$$
\begin{aligned}
& u_{0 k}(t):=\frac{2^{(k+1)}}{\sqrt{3} f(t)} \mathrm{e}^{-k t} \cosh ^{k} t_{2} F_{1}\left(k+3 / 2, k ;-1 / 2 ;-\mathrm{e}^{-2 t}\right), \\
& v_{0 k}(t):=-\frac{2^{(k+1)}}{\sqrt{3} f(t)} \mathrm{e}^{-(k+3) t} \cosh ^{k} t_{2} F_{1}\left(k+3 / 2, k+3 ; 5 / 2 ;-\mathrm{e}^{-2 t}\right),
\end{aligned}
$$

where ${ }_{2} F_{1}(a, b ; c ; z)$ are hypergeometric functions [30]. In this way, we get a very convenient expression for the symplectic structure (3.8) that will be our starting point for the quantization of the system

$$
\begin{aligned}
\Omega\left(\zeta_{1}, \zeta_{2}\right) & =i \sum_{k=0}^{\infty} \sum_{l=0}^{k} \sum_{m=-l}^{l}\left(\bar{a}_{k l m}^{(1)} a_{k l m}^{(2)}-\bar{a}_{k l m}^{(2)} a_{k l m}^{(1)}\right), \\
\text { where } \zeta_{\alpha} & =\sum_{k=0}^{\infty} \sum_{l=0}^{k} \sum_{m=-l}^{l}\left(a_{k l m}^{(\alpha)} \phi_{k l m}+\bar{a}_{k l m}^{(\alpha)} \bar{\phi}_{k l m}\right), \quad \alpha=1,2 .
\end{aligned}
$$

\section{QUANTUM HILBERT SPACE}

We will review in this section the Fock quantization technique based on the covariant phase space of the model. It is well known [31] that for a system of a finite number of uncoupled quantum harmonic oscillators this procedure provides a quantum theory unitarily equivalent to the usual tensor product of one-particle Hilbert spaces. However, for the case of a system of infinitely many uncoupled quantum harmonic oscillators, the tensor product of the infinite one particle Hilbert spaces gives rise to a non-separable Hilbert space, as well as reducible representations of the canonical commutation relations. For these reasons the Fock quantization introduced below provides a better approach to deal with the infinitely many degrees of freedom present in our model. 


\section{A. One particle Hilbert space and its Fock space}

Let $\mathcal{S}_{\mathbb{C}}$ be the complexification of the space of solutions $\mathcal{S}$ to the equation of motion (3.7). Every vector $Z \in \mathcal{S}_{\mathbb{C}}$ can be expressed as

$$
Z(t, s)=\sum_{k=0}^{\infty} \sum_{l=0}^{k} \sum_{m=-l}^{l}\left(a_{k l m} \phi_{k l m}(t, s)+b_{k l m} \bar{\phi}_{k l m}(t, s)\right), \quad a_{k l m}, b_{k l m} \in \mathbb{C} .
$$

Let us extend $\Omega$ to $\mathcal{S}_{\mathbb{C}}$ by complex linearity in each variable

$$
\Omega_{\mathbb{C}}\left(Z_{1}, Z_{2}\right)=i \sum_{k=0}^{\infty} \sum_{l=0}^{k} \sum_{m=-l}^{l}\left(b_{k l m}^{(1)} a_{k l m}^{(2)}-b_{k l m}^{(2)} a_{k l m}^{(1)}\right)
$$

and consider the sesquilinear map $\langle\cdot, \cdot\rangle: \mathcal{S}_{\mathbb{C}} \times \mathcal{S}_{\mathbb{C}} \rightarrow \mathbb{R}$ defined by

$$
\left\langle Z_{1}, Z_{2}\right\rangle:=-i \Omega_{\mathbb{C}}\left(\bar{Z}_{1}, Z_{2}\right)=\sum_{k=0}^{\infty} \sum_{l=0}^{k} \sum_{m=-l}^{l}\left(\bar{a}_{k l m}^{(1)} a_{k l m}^{(2)}-\bar{b}_{k l m}^{(1)} b_{k l m}^{(2)}\right) .
$$

This map satisfies all the properties of an inner product on $\mathcal{S}_{\mathbb{C}}$ except that it fails to be positive definite. However by considering the Lagrangian subspaces

$$
\begin{aligned}
& \mathbf{P}:=\left\{Z \in \mathcal{S}_{\mathbb{C}}: Z=\sum_{k=0}^{\infty} \sum_{l=0}^{k} \sum_{m=-l}^{l} a_{k l m} \phi_{k l m}\right\} \\
& \overline{\mathbf{P}}:=\left\{Z \in \mathcal{S}_{\mathbb{C}}: Z=\sum_{k=0}^{\infty} \sum_{l=0}^{k} \sum_{m=-l}^{l} b_{k l m} \bar{\phi}_{k l m}\right\}
\end{aligned}
$$

it is possible to decompose $\mathcal{S}_{\mathbb{C}}=\mathbf{P} \oplus \overline{\mathbf{P}}$ thus obtaining a positive definite restriction $\left.\langle\cdot, \cdot\rangle\right|_{\mathbf{P}}$. The separable and infinite-dimensional one-particle Hilbert space $\mathcal{H}_{\mathbf{P}} \cong \ell_{2}$ is obtained by Cauchy completion

$$
\mathcal{H}_{\mathbf{P}}:=\overline{\left(\mathbf{P},\left.\langle\cdot, \cdot\rangle\right|_{\mathbf{P}}\right)}\langle\cdot \cdot \cdot\rangle_{\mathbf{P}}=\left\{Z: Z=\sum_{k l m} a_{k l m} \phi_{k l m}, a_{k l m} \in \mathbb{C}, \sum_{k l m}\left|a_{k l m}\right|^{2}<\infty\right\} .
$$

The set $\left\{\phi_{k l m}\right\}$ becomes a countable orthonormal basis of $\mathcal{H}_{\mathbf{P}}$. The Hilbert space of the quantum field theory $\mathcal{F}_{s}\left(\mathcal{H}_{\mathbf{P}}\right)$ is then the symmetric Fock space

$$
\mathcal{F}_{s}\left(\mathcal{H}_{\mathbf{P}}\right):=\bigoplus_{n=0}^{\infty} \mathcal{H}_{\mathbf{P}}^{\otimes_{s}^{n}}
$$

where $\mathcal{H}_{\mathbf{P}}^{\otimes_{s}^{n}}$ denotes the Hilbert space of all $n$-th rank symmetric tensors over $\mathcal{H}_{\mathbf{P}}$.

Notice that every choice (4.4)-(4.5) of the Lagrangian subspaces $\mathbf{P}$ and $\overline{\mathbf{P}}$ corresponds to the specification of a complex structure $J_{\mathbf{P}}$ on the space of solutions $\mathcal{S}$. Indeed, due to the fact that $\mathbf{P} \cap \overline{\mathbf{P}}=\{0\}$, it follows that $\mathcal{S}_{\mathbb{C}}=\mathbf{P} \oplus \overline{\mathbf{P}}$, and hence any vector $\zeta \in \mathcal{S}$ can be uniquely decomposed as $\zeta=Z+\bar{Z}$, with $Z \in \mathbf{P}, \bar{Z} \in \overline{\mathbf{P}}$. Then, given $\mathbf{P}$ and $\overline{\mathbf{P}}$, we 
can define the complex structure $J_{\mathbf{P}}: \mathcal{S} \rightarrow \mathcal{S}$ by $J_{\mathbf{P}} \zeta:=i Z-i \bar{Z}$. This map is a linear canonical transformation on $\Gamma=(\mathcal{S}, \Omega)$-i.e. $J_{\mathbf{P}}$ on $\mathcal{S}$ is compatible with $\Omega$ - such that $J_{\mathbf{P}}^{2}=J_{\mathbf{P}} \circ J_{\mathbf{P}}=-\operatorname{Id}_{\mathcal{S}}$ and the formula

$$
\mu_{J_{\mathbf{P}}}\left(\zeta_{1}, \zeta_{2}\right):=\frac{1}{2} \Omega\left(J_{\mathbf{P}} \zeta_{1}, \zeta_{2}\right)
$$

defines a positive definite bilinear symmetric form on $\mathcal{S}$. We conclude then that the sesquilinear map

$$
\left\langle\zeta_{1}, \zeta_{2}\right\rangle_{J_{\mathbf{P}}}:=\mu_{J_{\mathbf{P}}}\left(\zeta_{1}, \zeta_{2}\right)-\frac{i}{2} \Omega\left(\zeta_{1}, \zeta_{2}\right)
$$

is an inner product on $\mathcal{S}_{J_{\mathrm{P}}}$ [32]. Here $\mathcal{S}_{J_{\mathrm{P}}}$ is the complex vector space $\mathcal{S}$ where, given any $\zeta \in \mathcal{S}$, the product by complex scalars $\mathbb{C} \ni z=x+i y, x, y \in \mathbb{R}$, is defined by the rule $z \cdot \zeta:=x \zeta+y J_{\mathbf{P}} \zeta$. In this context, the one-particle Hilbert space $\mathcal{H}_{\mathbf{P}}$ is given by the Cauchy completion of the Euclidean space $\left(S_{J_{\mathbf{P}}},\langle\cdot, \cdot\rangle_{J_{\mathbf{P}}}\right)$. It is straightforward to check that the Cauchy completions of $(\mathbf{P},\langle\cdot, \cdot\rangle)$ and $\left(S_{J_{\mathbf{P}}},\langle\cdot, \cdot\rangle_{J_{\mathbf{P}}}\right)$ are isomorphic: the $\mathbb{C}$-linear map $\kappa: S_{J_{\mathbf{P}}} \rightarrow \mathbf{P}$ such that $\kappa(\zeta)=Z, \zeta \in \mathcal{S}_{J_{\mathbf{P}}}, Z \in \mathbf{P}$, defines a unitary transformation of $\mathcal{S}_{J_{\mathbf{P}}}$ onto $\mathbf{P}$, i.e., $\left\langle\zeta_{1}, \zeta_{2}\right\rangle_{J_{\mathbf{P}}}=\left\langle\kappa\left(\zeta_{1}\right), \kappa\left(\zeta_{2}\right)\right\rangle=\left\langle Z_{1}, Z_{2}\right\rangle, \forall \zeta_{1}, \zeta_{2} \in \mathcal{S}_{J_{\mathbf{P}}}$

\section{B. Complex structures and mode decomposition}

In practice, the definition of the complex structure $J_{\mathbf{P}}$ is complete once we are given a family of complex functions $\left\{y_{k}\right\}$ satisfying (3.18) and (3.20). In this case, we can construct an orthonormal basis $\left\{\phi_{k l m}=y_{k} Y_{k l m}\right\}$ of the one particle Hilbert space $\mathcal{H}_{\mathbf{P}}$ and define $J_{\mathbf{P}}$ by imposing that the complex structure is diagonalized in $\mathcal{S}_{\mathbb{C}}$

$$
J_{\mathbf{P}} \phi_{k l m}=i \phi_{k l m}, \quad J_{\mathbf{P}} \bar{\phi}_{k l m}=-i \bar{\phi}_{k l m} .
$$

As we will see in the next section, these choices represent all the possibilities for the $S O(4)$ invariant complex structures in $\mathcal{S}$. Here we discuss the freedom in the choice of the normalized set $\left\{y_{k}\right\}$. To this end, let us fix once and for all a family $\left\{y_{0 k}=u_{0 k}+i v_{0 k}\right\}$ verifying the normalization condition

$$
\bar{y}_{0 k}(t) \dot{y}_{0 k}(t)-\dot{\bar{y}}_{0 k}(t) y_{0 k}(t)=\frac{i}{f^{2}(t) \cosh ^{3}(t)} .
$$

For example by considering the pairs $\left(u_{0 k}, v_{0 k}\right)$ given by (3.21). Then, any other normalized set $\left\{y_{k}\right\}$ can be expressed in terms of $u_{0 k}$ and $v_{0 k}$ as

$$
y_{k}(t)=\alpha_{k} u_{0 k}(t)+\beta_{k} v_{0 k}(t)+i\left(\gamma_{k} u_{0 k}(t)+\delta_{k} v_{0 k}(t)\right),
$$

where the real coefficients $\alpha_{k}, \beta_{k}, \gamma_{k}, \delta_{k}$ must satisfy

$$
\operatorname{det}\left(\begin{array}{cc}
\alpha_{k} & \beta_{k} \\
\gamma_{k} & \delta_{k}
\end{array}\right)=1 \Leftrightarrow\left(\begin{array}{cc}
\alpha_{k} & \beta_{k} \\
\gamma_{k} & \delta_{k}
\end{array}\right) \in S L(2, \mathbb{R}), \forall k \in \mathbb{N}_{0}
$$

It is well known that $S L\left(2, \mathbb{R}\right.$ ) is bijective (as a set) to $\mathbb{S}^{1} \times \mathbb{R}^{2}$, in the sense that any element of $S L(2, \mathbb{R})$ can be uniquely decomposed as a product of a rotation and an upper triangular 
matrix with unit determinant

$$
S L(2, \mathbb{R}) \ni\left(\begin{array}{ll}
\alpha_{k} & \beta_{k} \\
\gamma_{k} & \delta_{k}
\end{array}\right)=\left(\begin{array}{cc}
\cos \theta_{k} & -\sin \theta_{k} \\
\sin \theta_{k} & \cos \theta_{k}
\end{array}\right)\left(\begin{array}{cc}
\rho_{k} & \nu_{k} \\
0 & \rho_{k}^{-1}
\end{array}\right),
$$

for a unique choice of $\rho_{k}>0, \nu_{k} \in \mathbb{R}, \theta_{k} \in[0,2 \pi)$. Hence, different choices of the triplet $\left(\rho_{k}, \nu_{k}, \theta_{k}\right)$ will, in principle, correspond to different complex structures on the space of solutions $\mathcal{S}$, defined through (4.9) where

$$
\begin{aligned}
y_{k}(t) & =\rho_{k} \cos \theta_{k} u_{0 k}(t)+\left(\nu_{k} \cos \theta_{k}-\rho_{k}^{-1} \sin \theta_{k}\right) v_{0 k}(t) \\
& +i\left(\rho_{k} \sin \theta_{k} u_{0 k}(t)+\left(\nu_{k} \sin \theta_{k}+\rho_{k}^{-1} \cos \theta_{k}\right) v_{0 k}(t)\right) .
\end{aligned}
$$

However, it is easy to show [15] that two different choices of the form $\left(\rho_{k}, \nu_{k}, \theta_{k}\right)$ and $\left(\rho_{k}, \nu_{k}, \tilde{\theta}_{k}\right)$ give rise to the same complex structure. Then, in the following, we will omit the angular part of $\left(\rho_{k}, \nu_{k}, \theta_{k}\right)$ by choosing $\theta_{k}=0$ in (4.12).

The complex structures defined trough $\left(\rho_{k}, \nu_{k}\right)$ will, in general, yield irreducible unitarily nonequivalent representations of the canonical commutation relations. The existence of many non-unitarily equivalent quantization is a well known property of any QFT in a generic curved space-time, and can be considered as a serious drawback to the formulation of the theory. Obviously, this is not the case for a system of finite number of degrees of freedom, where the Stone-von Neumann theorem can be applied [33]: for any Lagrangian subspace $\mathbf{P}$ one obtains a quantum theory unitarily equivalent to the usual tensor product construction. Also, for the case of a massless scalar field evolving in a fixed stationary space-time, there exists a preferred choice of Lagrangian subspace by virtue of the time translation symmetry [31]. In our case, in absence of this symmetry, no natural, preferred election of $\mathbf{P}$ is available; in other words, due to the time-dependence of the Hamiltonian (3.11) the solutions of $\mathcal{S}$ do not oscillate harmonically, and thus it is not possible to uniquely define subspaces of positive and negative frequency solutions.

\section{C. $S O(4)$-invariant complex structures}

Consider some specific basis $\left\{\phi_{0 k l m}\right\}$ of $\mathcal{H}_{\mathbf{P}}$ and the corresponding splitting of the complexified solution space $\mathcal{S}_{\mathbb{C}}=\mathbf{P}_{0} \oplus \overline{\mathbf{P}}_{0}$. For practical purposes, let us denote

$$
\begin{aligned}
& \mathbf{P}_{1}:=\mathbf{P}_{0}=\bigoplus_{k=0}^{\infty} \mathbf{P}_{1}^{k}, \mathbf{P}_{1}^{k}:=\operatorname{span}\left\{y_{0 k}\right\} \otimes \operatorname{span}\left\{Y_{k l m} \mid l \in\{0,1, \ldots, k\}, m \in\{-l, \ldots, l\}\right\}, \\
& \mathbf{P}_{2}:=\overline{\mathbf{P}}_{0}=\bigoplus_{k=0}^{\infty} \mathbf{P}_{2}^{k}, \quad \mathbf{P}_{2}^{k}:=\operatorname{span}\left\{\bar{y}_{0 k}\right\} \otimes \operatorname{span}\left\{Y_{k l m} \mid l \in\{0,1, \ldots, k\}, m \in\{-l, \ldots, l\}\right\} .
\end{aligned}
$$

The elements $\phi_{a} \in \mathbf{P}_{a}, a=1,2$, are complex functions $\phi_{a}(t, s)$ defined on $\mathbb{R} \times \mathbb{S}^{3}$. The natural representation $D_{a}$ of $S O(4)$ in $\mathbf{P}_{a}$ is then defined by $\left(D_{a}(g) \phi\right)(t, s)=\phi\left(t, g^{-1} \cdot s\right)$, where $g^{-1} \cdot s$ denotes the action of the rotation $g^{-1} \in S O(4)$ on the point $s \in \mathbb{S}^{3}$. This allows us to write the representation of $S O(4)$ in $\mathcal{S}_{\mathbb{C}}=\mathbf{P}_{1} \oplus \mathbf{P}_{2}$ in matrix form

$$
D(g)=\left(\begin{array}{cc}
D_{1}(g) & 0 \\
0 & D_{2}(g)
\end{array}\right), \quad g \in S O(4),
$$


in terms of the representations $\left(D_{a}, \mathbf{P}_{a}\right)$. In particular, we are interested in characterizing the complex structures $J$ defined on the solution space $\mathcal{S}$ invariant under the $S O(4)$ symmetry of the round 3-sphere $\mathbb{S}^{3}$. Using the previous notation, this implies

$$
D(g) J=J D(g) \Leftrightarrow\left(\begin{array}{ll}
J_{11} D_{1}(g) & J_{12} D_{2}(g) \\
J_{21} D_{1}(g) & J_{22} D_{2}(g)
\end{array}\right)=\left(\begin{array}{cc}
D_{1}(g) J_{11} & D_{1}(g) J_{12} \\
D_{2}(g) J_{21} & D_{2}(g) J_{22}
\end{array}\right), \forall g \in S O(4),
$$

in terms of $\mathbb{C}$-linear maps $J_{a b}: \mathbf{P}_{b} \rightarrow \mathbf{P}_{a}, a, b \in\{1,2\}$. The same arguments used in [15] with the aim of identifying the $S O(3)$-invariant complex structures for the Gowdy $\mathbb{S}^{1} \times \mathbb{S}^{2}$ and $\mathbb{S}^{3}$ models show now that the general form of a $\mathbb{R}$-linear operator $J$ satisfying (4.14) is given by

$$
J=\bigoplus_{k=0}^{\infty}\left(\begin{array}{ccc}
j_{11}^{k} I_{11}^{k} & j_{12}^{k} I_{12}^{k} \\
\bar{j}_{12}^{k} I_{21}^{k} & \bar{j}_{11}^{k} I_{22}^{k}
\end{array}\right)
$$

where $I_{a a}^{k}$ denotes the identity operator in $\mathbf{P}_{a}^{k}$ and the linear operators $I_{a b}^{k}: \mathbf{P}_{b}^{k} \rightarrow \mathbf{P}_{a}^{k}$ act according to $I_{12}^{k}\left(\bar{y}_{0 k} \otimes v\right)=y_{0 k} \otimes v$ and $I_{21}^{k}\left(y_{0 k} \otimes v\right)=\bar{y}_{0 k} \otimes v$. Finally, due to the extra condition $J^{2}=-\mathrm{Id}_{\mathbb{S}}$, defining $J$ as a complex structure, the complex coefficients $j_{11}^{k}$ and $j_{12}^{k}$ should verify

$$
\left|\jmath_{11}^{k}\right|^{2}-\left|\jmath_{12}^{k}\right|^{2}=1, \quad \jmath_{11}^{k} \in i \mathbb{R} \backslash\{0\}, \quad \jmath_{12}^{k} \in \mathbb{C} .
$$

According to this result, it suffices to take $\jmath_{11}^{k}=i$ and $\jmath_{12}^{k}=0, \forall k \in \mathbb{N}_{0}$, to conclude that all the complex structures $J_{\mathbf{P}}$ defined in section IVB are $S O(4)$-invariant. It is also clear that any $S O(4)$-invariant complex structure, characterized by the pairs $\left(j_{11}^{k}, j_{12}^{k}\right)$ verifying (4.15), has an associated Lagrangian subspace $\mathbf{P}$ defined by the set $\left\{y_{k}=\rho_{k} u_{0 k}+\left(\nu_{k}+i \rho_{k}^{-1}\right) v_{0 k}\right\}$, with the coefficients $\left(\rho_{k}, \nu_{k}\right)$ related to $\left(j_{11}^{k}, j_{12}^{k}\right)$ by

$$
j_{11}^{k}=\frac{i}{2}\left(\nu_{k}^{2}+\rho_{k}^{-2}+\rho_{k}^{2}\right), \quad j_{12}^{k}=-\rho_{k} \nu_{k}+\frac{i}{2}\left(\nu_{k}^{2}+\rho_{k}^{-2}-\rho_{k}^{2}\right) .
$$

\section{Canonical quantum field operators}

The canonical field operators associated to a given time $t \in \mathbb{R}$ are defined as operatorvaluated distributions on $\mathbb{S}^{3}$

$$
\begin{aligned}
\hat{Q}(t, s)=\sum_{k=0}^{\infty} \sum_{l=0}^{k} \sum_{m=-l}^{l}\left(y_{k}(t) Y_{k l m}(t) \hat{a}_{k l m}+\bar{y}_{k}(t) \bar{Y}_{k l m}(s) \hat{a}_{k l m}^{\dagger}\right), \\
\hat{P}(t, s)=\sum_{k=0}^{\infty} \sum_{l=0}^{k} \sum_{m=-l}^{l}\left(\left[f^{2}(t) \cosh ^{3} t \dot{y}_{k}(t)+f(t) \dot{f}(t) \cosh ^{3} t y_{k}(t)\right] Y_{k l m}(t) \hat{a}_{k l m}\right. \\
\left.+\left[f^{2}(t) \cosh ^{3} t \dot{\bar{y}}_{k}(t)+f(t) \dot{f}(t) \cosh ^{3} t \bar{y}_{k}(t)\right] \bar{Y}_{k l m}(s) \hat{a}_{k l m}^{\dagger}\right) .
\end{aligned}
$$

In practice, these expressions can be obtained by formally promoting the Fourier coefficients in (3.10) to the creation and annihilation operators $-\hat{a}_{k l m}^{\dagger}$ and $\hat{a}_{k l m}$, respectivelyassociated to the basic vectors $\phi_{k l m} \in \mathcal{H}_{\mathbf{P}}$. Given any pair of smooth real-valued functions on the 3 -sphere $g_{1}, g_{2} \in C^{\infty}\left(\mathbb{S}^{3} ; \mathbb{R}\right)$, these distributions define canonical field operators $\left(\hat{Q}\left[g_{1}\right](t), \hat{P}\left[g_{2}\right](t)\right)$ obtained by multiplying the formal expressions (4.16) and (4.17) by $g_{1}$ and $g_{2}$, respectively, and integrating over the round 3 -sphere $\mathbb{S}^{3}$. In the next section we will 
study the behavior of these canonical operators in $t$. In particular we will consider if it is possible to choose the conformal factor $f$ and the complex structure, defined through $\left\{y_{k}\right\}$, in such a way that the functional dependence in $t$ of $(\hat{Q}(t, s), \hat{P}(t, s))$ can be obtained by the action of an unitary operator in the Fock space. As we will see, this is not the case.

\section{CLASSICAL DYNAMICS AND UNITARY QUANTUM EVOLUTION}

Classical time evolution from the embedding $\imath_{t_{0}}\left(\mathbb{S}^{3}\right) \subset \mathbb{R} \times \mathbb{S}^{3}$ to $\imath_{t_{1}}\left(\mathbb{S}^{3}\right) \subset \mathbb{R} \times \mathbb{S}^{3}$ is implemented on the canonical phase space $\Upsilon$ by the symplectic transformation $\tau_{\left(t_{0}, t_{1}\right)}: \Upsilon \rightarrow$ $\Upsilon$ defined as

$$
\tau_{\left(t_{0}, t_{1}\right)}:=\mathfrak{J}_{t_{1}} \circ \mathfrak{J}_{t_{0}}^{-1}
$$

in terms of the symplectic maps $\mathfrak{J}_{t}$ introduced in $(\underline{3.10})$ and their inverses $\mathfrak{J}_{t}^{-1}: \Upsilon \rightarrow \Gamma$, $\zeta=\mathfrak{J}_{t}^{-1}(Q, P)$. The maps $\mathfrak{J}_{t}^{-1}$ can be easily computed in terms of the Fourier coefficients (3.17) of $\zeta$ as

$$
a_{k l m}(t)=i\left(f(t) \dot{\bar{y}}_{k}(t)+\dot{f}(t) \bar{y}_{k}(t)\right) f(t) \cosh ^{3} t \int_{\mathbb{S}^{3}}|\gamma|^{1 / 2} Y_{k l m} Q-i \bar{y}_{k}(t) \int_{\mathbb{S}^{3}}|\gamma|^{1 / 2} Y_{k l m} P .
$$

The operator $\tau_{\left(t_{0}, t_{1}\right)}$ acts as follows: (i) first, it takes initial Cauchy data on $\imath_{t_{0}}\left(\mathbb{S}^{3}\right)$, (ii) evolves them to the corresponding solution in $\mathcal{S}$, and (iii) finds the Cauchy data induced by this solution on $\imath_{t_{1}}\left(\mathbb{S}^{3}\right)$. On the other hand, time evolution can also be viewed as a symplectic transformation on the covariant phase space, $\mathcal{T}_{\left(t_{0}, t_{1}\right)}: \Gamma \rightarrow \Gamma$, defined by

$$
\mathcal{T}_{\left(t_{0}, t_{1}\right)}:=\mathfrak{J}_{t_{1}}^{-1} \circ \tau_{\left(t_{0}, t_{1}\right)} \circ \mathfrak{J}_{t_{1}}=\mathfrak{J}_{t_{0}}^{-1} \circ \mathfrak{J}_{t_{1}}
$$

that (i) takes a solution of $\mathcal{S}$, (ii) finds the induced Cauchy data on $\imath_{t_{1}}\left(\mathbb{S}^{3}\right)$, and (iii) takes that data as initial data on $\imath_{t_{0}}\left(\mathbb{S}^{3}\right)$, finding finally the corresponding solution. In our case, combining (3.10) and (5.1), it is straightforward to check that, whenever

$$
\zeta=\sum_{k=0}^{\infty} \sum_{l=0}^{k} \sum_{m=-l}^{l}\left(a_{k l m} \phi_{k l m}+\bar{a}_{k l m} \bar{\phi}_{k l m}\right),
$$

the action of the operator $\mathcal{T}_{\left(t_{0}, t_{1}\right)}$ is given by

$$
\mathcal{T}_{\left(t_{0}, t_{1}\right)} \zeta=\sum_{k=0}^{\infty} \sum_{l=0}^{k} \sum_{m=-l}^{l}\left(\mathfrak{a}_{k l m}\left(t_{0}, t_{1}\right) \phi_{k l m}+\overline{\mathfrak{a}}_{k l m}\left(t_{0}, t_{1}\right) \bar{\phi}_{k l m}\right)
$$

where

$$
\begin{aligned}
\mathfrak{a}_{k l m}\left(t_{0}, t_{1}\right) & :=i\left(\frac{f\left(t_{0}\right)}{f\left(t_{1}\right)} \cosh ^{3}\left(t_{0}\right) \dot{\bar{z}}_{k}\left(t_{0}\right) z_{k}\left(t_{1}\right)-\frac{f\left(t_{1}\right)}{f\left(t_{0}\right)} \cosh ^{3}\left(t_{1}\right) \dot{z}_{k}\left(t_{1}\right) \bar{z}_{k}\left(t_{0}\right)\right) a_{k l m} \\
& +i\left(\frac{f\left(t_{0}\right)}{f\left(t_{1}\right)} \cosh ^{3}\left(t_{0}\right) \dot{\bar{z}}_{k}\left(t_{0}\right) \bar{z}_{k}\left(t_{1}\right)-\frac{f\left(t_{1}\right)}{f\left(t_{0}\right)} \cosh ^{3}\left(t_{1}\right) \dot{\bar{z}}_{k}\left(t_{1}\right) \bar{z}_{k}\left(t_{0}\right)\right) \bar{a}_{k m l} .
\end{aligned}
$$


Here we have introduced the functions

$$
z_{k}(t):=f(t) y_{k}(t)
$$

that will allow us to use a more economical notation in what follows.

\section{A. Unitary implementation of quantum dynamics}

Let us consider now the quantum counterpart of the classical time evolution. Taking a $S O(4)$-invariant complex structure $J_{\mathbf{P}}$ and using the theory of unitary implementation of symplectic transformations [1, 31], the time evolution $\mathcal{T}_{\left(t_{0}, t_{1}\right)}$ defined in (5.2) is unitarily implementable on the Fock space $\mathcal{F}_{s}\left(\mathcal{H}_{P}\right)$, i.e., there exists a unitary operator $\hat{U}\left(t_{0}, t_{1}\right)$ : $\mathcal{F}_{s}\left(\mathcal{H}_{\mathcal{P}}\right) \rightarrow \mathcal{F}_{s}\left(\mathcal{H}_{P}\right)$ such that

$$
\hat{U}^{-1}\left(t_{0}, t_{1}\right) \hat{Q}\left(t_{0}, s\right) \hat{U}\left(t_{0}, t_{1}\right)=\hat{Q}\left(t_{1}, s\right), \hat{U}^{-1}\left(t_{0}, t_{1}\right) \hat{P}\left(t_{0}, s\right) \hat{U}\left(t_{0}, t_{1}\right)=\hat{P}\left(t_{1}, s\right),
$$

if and only if $J_{\mathbf{P}}-\mathcal{T}_{\left(t_{0}, t_{1}\right)}^{-1} \circ J_{\mathbf{P}} \circ \mathcal{T}_{\left(t_{0}, t_{1}\right)}$ is Hilbert-Schmidt. This is equivalent to demand that

$$
\sum_{k=0}^{\infty}(k+1)^{2}\left|\beta_{k}\left(t_{0}, t_{1} \mid z_{k}, f\right)\right|^{2}<\infty
$$

where

$$
\beta_{k}\left(t_{0}, t_{1} \mid z_{k}, f\right):=\frac{f\left(t_{0}\right)}{f\left(t_{1}\right)} \cosh ^{3}\left(t_{0}\right) \dot{z}_{k}\left(t_{0}\right) z_{k}\left(t_{1}\right)-\frac{f\left(t_{1}\right)}{f\left(t_{0}\right)} \cosh ^{3}\left(t_{1}\right) \dot{z}_{k}\left(t_{1}\right) z_{k}\left(t_{0}\right) .
$$

Notice that the square summability of the series (5.5) depends only on its ultraviolet behavior. In particular, the zero mode corresponding to $k=0$ plays no role in this context. As we pointed out in subsection IVC, the $S O(4)$-invariant complex structures differ from each other just in the pair $\left(\rho_{k}, \nu_{k}\right)$ which appears inside of each $z_{k}=f y_{k}$. Then we will consider the complex structures induced by choosing $z_{k}=f y_{k}$ of the form

$$
z_{k}(t)=\rho_{k} f(t) u_{0 k}(t)+\left(\nu_{k}+i \rho_{k}^{-1}\right) f(t) v_{0 k}(t)=\rho_{k} \tilde{u}_{0 k}(t)+\left(\nu_{k}+i \rho_{k}^{-1}\right) \tilde{v}_{0 k}(t)
$$


with

$$
\begin{aligned}
\tilde{u}_{0 k}(t):= & f(t) u_{0 k}(t)=\frac{2^{(k+1)}}{\sqrt{3}} \cosh ^{k}(t) \mathrm{e}^{-k t}{ }_{2} F_{1}\left(k+3 / 2, k ;-1 / 2 ;-\mathrm{e}^{-2 t}\right) \\
\tilde{v}_{0 k}(t):= & f(t) v_{0 k}(t)=-\frac{2^{(k+1)}}{\sqrt{3}} \cosh ^{k}(t) \mathrm{e}^{-(k+3) t}{ }_{2} F_{1}\left(k+3 / 2, k+3 ; 5 / 2 ;-\mathrm{e}^{-2 t}\right) \\
\dot{\tilde{u}}_{0 k}(t)= & -\frac{2^{(k+1)}}{\sqrt{3}} k \cosh ^{k-1}(t) \mathrm{e}^{-(k+2) t}\left(\mathrm{e}^{t}{ }_{2} F_{1}\left(k, k+3 / 2 ;-1 / 2 ;-\mathrm{e}^{-2 t}\right)\right. \\
& \left.+2(2 k+3) \cosh (t){ }_{2} F_{1}\left(k+1, k+5 / 2 ; 1 / 2 ;-\mathrm{e}^{-2 t}\right)\right) \\
\dot{\tilde{v}}_{0 k}(t)= & \frac{2^{k}}{5 \sqrt{3}} \cosh ^{k-1}(t) \mathrm{e}^{-(k+5) t}\left(5\left(3+2 k+3 \mathrm{e}^{2 t}\right) \mathrm{e}^{t}{ }_{2} F_{1}\left(k+3 / 2, k+3 ; 5 / 2 ;-\mathrm{e}^{-2 t}\right)\right. \\
& \left.-4(k+3)(2 k+3) \cosh (t){ }_{2} F_{1}\left(k+5 / 2, k+4 ; 7 / 2 ;-\mathrm{e}^{-2 t}\right)\right)
\end{aligned}
$$

Taking into account the asymptotic behaviors (valid for $\lambda \rightarrow+\infty, \alpha, \beta \in \mathbb{R}, 0<z<1$ )

$$
\begin{aligned}
{ }_{2} F_{1}\left(\lambda+\alpha, \lambda+\beta ; \frac{5}{2} ;-z\right) & \sim-\frac{3}{4 z \lambda^{2}}(1+z)^{\frac{5}{4}-\lambda-\frac{\alpha+\beta}{2}} \cos \Theta(\lambda, \alpha+\beta-2 ; z), \\
{ }_{2} F_{1}\left(\lambda+\alpha, \lambda+\beta ;-\frac{1}{2} ;-z\right) & \sim 2 \lambda \sqrt{z}(1+z)^{-\frac{1}{4}-\lambda-\frac{\alpha+\beta}{2}} \sin \Theta(\lambda, \alpha+\beta+1 ; z), \\
{ }_{2} F_{1}\left(\lambda+\alpha, \lambda+\beta ; \frac{1}{2} ;-z\right) & \sim(1+z)^{\frac{1}{4}-\lambda-\frac{\alpha+\beta}{2}} \cos \Theta(\lambda, \alpha+\beta ; z), \\
{ }_{2} F_{1}\left(\lambda+\alpha, \lambda+\beta ; \frac{7}{2} ;-z\right) & \sim-\frac{15}{8} z^{-3 / 2} \lambda^{-3}(1+z)^{\frac{7}{4}-\lambda-\frac{\alpha+\beta}{2}} \sin \Theta(\lambda, \alpha+\beta-3 ; z),
\end{aligned}
$$

with

$$
\Theta(\lambda, \gamma ; z):=(2 \lambda+\gamma) \arctan \sqrt{z}-\arctan \frac{\sqrt{z}}{1+\sqrt{1+z}},
$$

we get that, for $k \rightarrow+\infty$,

$$
\begin{aligned}
\tilde{u}_{0 k}(t) \sim & \frac{2}{\sqrt{3}} \frac{k}{\cosh (t)} \sin \Theta\left(k, 5 / 2 ; \mathrm{e}^{-2 t}\right) \\
\tilde{v}_{0 k}(t) \sim & \frac{\sqrt{3}}{4 k^{2}} \frac{1}{\cosh (t)} \cos \Theta\left(k, 5 / 2 ; \mathrm{e}^{-2 t}\right) \\
\dot{\tilde{u}}_{0 k}(t) \sim & -\frac{2 k^{2}}{\sqrt{3}} \frac{\mathrm{e}^{-2 t}}{\cosh (t)}\left(2\left(1+\mathrm{e}^{-2 t}\right)^{-1} \sin \Theta\left(k, 5 / 2 ; \mathrm{e}^{-2 t}\right)\right. \\
& \left.+4 \cosh (t)\left(1+\mathrm{e}^{-2 t}\right)^{-\frac{3}{2}} \cos \Theta\left(k, 7 / 2 ; \mathrm{e}^{-2 t}\right)\right) \\
\dot{\tilde{v}}_{0 k}(t) \sim & -\frac{\sqrt{3}}{2 k} \frac{\mathrm{e}^{-2 t}}{\cosh (t)}\left(\left(1+\mathrm{e}^{-2 t}\right)^{-1} \cos \Theta\left(k, 5 / 2 ; \mathrm{e}^{-2 t}\right)\right. \\
& \left.-2 \cosh (t)\left(1+\mathrm{e}^{-2 t}\right)^{-\frac{3}{2}} \sin \Theta\left(k, 7 / 2 ; \mathrm{e}^{-2 t}\right)\right) .
\end{aligned}
$$


These asymptotic expansions can be obtained by using the steepest descent method in a multi-variable integral representation of the hypergeometric function ${ }_{2} F_{1}$ [34]. Since

$$
\begin{aligned}
\operatorname{Im}\left[\beta_{k}\left(t_{0}, t_{1} \mid z_{k}, f\right)\right] & =\frac{f\left(t_{0}\right)}{f\left(t_{1}\right)} \cosh ^{3}\left(t_{0}\right)\left(\dot{\tilde{u}}_{0 k}\left(t_{0}\right) \tilde{v}_{0 k}\left(t_{1}\right)+\dot{\tilde{v}}_{0 k}\left(t_{0}\right) \tilde{u}_{0 k}\left(t_{1}\right)+2 \nu_{k} \rho_{k}^{-1} \dot{\tilde{v}}_{0 k}\left(t_{0}\right) \tilde{v}_{0 k}\left(t_{1}\right)\right) \\
& -\frac{f\left(t_{1}\right)}{f\left(t_{0}\right)} \cosh ^{3}\left(t_{1}\right)\left(\dot{\tilde{u}}_{0 k}\left(t_{1}\right) \tilde{v}_{0 k}\left(t_{0}\right)+\dot{\tilde{v}}_{0 k}\left(t_{1}\right) \tilde{u}_{0 k}\left(t_{0}\right)+2 \nu_{k} \rho_{k}^{-1} \dot{\tilde{v}}_{0 k}\left(t_{1}\right) \tilde{v}_{0 k}\left(t_{0}\right)\right),
\end{aligned}
$$

it is straightforward to show that, irrespective of the function $f>0$ and the complex structure parameterized by $\left(\rho_{k}, \nu_{k}\right)$,

$$
\lim _{k \rightarrow \infty} \operatorname{Im}\left[\beta_{k}\left(t_{0}, t_{1} \mid z_{k}, f\right)\right] \neq 0 .
$$

Hence, it is not possible to find a conformal factor $f$ and a complex structure $J_{\mathbf{P}}$ such that the dynamics can be unitarily implemented on $\mathcal{F}_{s}\left(\mathcal{H}_{\mathbf{P}}\right)$.

\section{CONCLUSIONS AND COMMENTS}

Some comments are in order now. First of all, by specializing the previous result to the choice $f(t)=1$, we have shown in section $\mathrm{VA}$ that it is impossible to find a Fock quantization of a minimally coupled massless scalar field $\phi$ propagating in de Sitter spacetime such that the quantum dynamics can be unitarily implemented. The only constraint that our Fock quantizations should satisfy is that they must be derived from a $S O(4)$ invariant complex structure on the space of classical solutions. The main result of the paper is that, in contrast with the Gowdy models, in the case of massless scalars in de Sitter space-time it is not possible to attain the quantum unitarity of the evolution by considering a spatially homogeneous field redefinition of the form $\zeta(t, s)=f^{-1}(t) \phi(t, s)$.

It is important to notice that for the $\mathbb{S}^{1} \times \mathbb{S}^{2}$ and $\mathbb{S}^{3}$ Gowdy models the conformal transformation that leads to a well behaved quantum evolution is the one that relates the background Gowdy metrics to the Einstein static universe [14, 15]. In the case of the de Sitter space, the transformation that connects the de Sitter metric to the Einstein static universe leads us to consider $f(t)=1 / \cosh (t)$. Now the scalar field $\zeta$ propagates in the Einstein static background universe but verifying a Klein-Gordon equation with a tachyonic time-dependent mass term. Explicitly the field $\zeta$ satisfies

$$
\ddot{\zeta}+\tanh (t) \dot{\zeta}-\operatorname{sech}^{2} t\left(\cosh (2 t)+\Delta_{\mathbb{S}^{3}}\right) \zeta=0 .
$$

Then, if we use the time coordinate $T=2 \arctan (\tanh (t / 2))$ defined in (2.3) in order to see the relation between the de Sitter and Einstein metrics, we get

$$
\partial_{T}^{2} \zeta-\left(\cosh (2 t)+\Delta_{\mathbb{S}^{3}}\right) \zeta=0 .
$$

It is clear that the time-dependent 'mass term' $\cosh (2 t)$ has the 'wrong sign'. Due to this fact, the modes (3.21) satisfy the harmonic oscillator equation with a time depende square frequency $k(k+2)-\cosh (2 t)$ whose sign, irrespectively of $k$, becomes negative when $t \rightarrow \pm \infty$ (or equivalently $T \rightarrow \pm \pi / 2$ ) . This introduces a non oscillatory behavior of these modes 
when $t \rightarrow \pm \infty$ that, at the end of the day, is one of the factors responsable of the failure of the unitarity condition.

It could appear at first sight that the failure of unitarity is also related to the presence of a first derivative term in the wave equation (6.1). This conclusion would be motivated by the Gowdy cases where the time variable usually used is such that the metric appears in the form $\stackrel{\circ}{g}_{a b}=-(\mathrm{d} t)_{a}(\mathrm{~d} t)_{b}+\gamma_{a b}^{\Sigma}$. In this way, the field $\zeta$ for which evolution is well defined verifies an equation $\stackrel{\circ}{g}^{a b} \nabla_{a} \dot{\nabla}_{b} \zeta=-\ddot{\zeta}+\Delta_{\Sigma} \zeta=\frac{1}{4}\left(1+\csc ^{2} t\right) \zeta$ without linear terms in $\dot{\zeta}$. On the contrary, for the 4 -dimensional de Sitter case, the metric $\stackrel{\circ}{g}_{a b}$ has the form $-(\mathrm{d} T)_{a}(\mathrm{~d} T)_{b}+\gamma_{a b}=-\dot{T}^{2}(\mathrm{~d} t)_{a}(\mathrm{~d} t)_{b}+\gamma_{a b}$, so that the field $\zeta$ verifies the equation (6.1) that involves a linear term in $\dot{\zeta}$. However, when the time coordinate $T=T(t)$ is used instead of $t$, the $\partial_{T} \zeta$-term disappear from the equation of motion. In conclusion, our results regarding unitarity do not depend on the way the evolution is parameterized.

Owing to the fact that the dynamical evolution is not unitarily implementable, one is faced with the non-existence of the usual Schrödinger picture. As far as models with a finite number of degrees of freedom is concerned, this situation is normally related to the lack of a suitable probabilistic interpretation of the theory. However, this is not necessarily the case for systems of infinitely many degrees of freedom, as it was pointed out for instance in [8] for the case of the Gowdy $\mathbb{T}^{3}$ model. Hence, the impossibility of implementing the dynamics in a unitary way is not an obstacle to consider this type of models as physically relevant. Indeed, concerning the de Sitter case, it is possible to address some physical sensible questions such as the cosmological constant problem [20] despite the absence of unitary dynamics.

In conclusion, the results discussed in this paper show that the method successfully used to obtain a unitary dynamics in the Fock quantization of the Gowdy models -based on a suitable redefinition of the scalar fields suggested by the conformal factor of the background metric- is not of general validity, not even in the context of highly symmetric space-time backgrounds.

\section{Acknowledgments}

The authors want to thank Fernando Barbero for many interesting discussions. Daniel Gómez Vergel acknowledges the support of the Spanish Research Council (CSIC) through a I3P research assistantship. This work is also supported by the Spanish MEC under the research grant FIS2005-05736-C03-02.

[1] D. Shale, Trans. Am. Math. Soc. 103, 149 (1962).

[2] A. D. Helfer, Class. Quant. Grav. 13, L129 (1996).

[3] C. G. Torre and M. Varadarajan, Class. Quant. Grav. 16, 2651 (1999).

[4] D. H. J. Cho and M. Varadarajan, Class. Quant. Grav. 23, 6115 (2006).

[5] M. Varadarajan, Phys. Rev. D75, 044018 (2007).

[6] M. Pierri, Int. J. Mod. Phys. D11, 135 (2002).

[7] A. Corichi, J. Cortez, and H. Quevedo, Int. J. Mod. Phys. D11, 1451 (2002).

[8] C. G. Torre, Phys. Rev. D66, 084017 (2002).

[9] A. Corichi, J. Cortez, and G. A. Mena Marugán, Phys. Rev. D73, 041502 (2006). 
[10] A. Corichi, J. Cortez, and G. A. Mena Marugán, Phys. Rev. D73, 084020 (2006).

[11] A. Corichi, J. Cortez, G. A. Mena Marugán, and J. M. Velhinho, Class. Quant. Grav. 23, 6301 (2006).

[12] J. Cortez, G. A. Mena Marugán, and J. M. Velhinho, Phys. Rev. D75, 084027 (2007).

[13] J. F. Barbero G., D. G. Vergel, and E. J. S. Villaseñor, Phys. Rev. D74, 024003 (2006).

[14] J. F. Barbero G., D. G. Vergel, and E. J. S. Villaseñor, Class. Quant. Grav. 24, 5945 (2007).

[15] J. F. Barbero G., D. G. Vergel, and E. J. S. Villaseñor. Quantum unitary evolution of linearly polarized $\mathbb{S}^{1} \times \mathbb{S}^{2}$ and $\mathbb{S}^{3}$ Gowdy models coupled to massless scalar fields. Class. Quant. Grav. (accepted for publication) arXiv:0711.1790.

[16] R. H. Gowdy, Phys. Rev. Lett. 27, 826 (1971).

[17] R. H. Gowdy, Ann. Phys. 83, 203 (1974).

[18] E. Angelopoulos, M. Flato, C. Fronsdal, and D. Sternheimer, Phys. Rev. D23, 1278 (1981).

[19] J. P. Gazeau, J. Renaud, and M. V. Takook, Class. Quant. Grav. 17, 1415 (2000).

[20] E. Mottola, Phys. Rev. D31, 754 (1985).

[21] E. Mottola, Phys. Rev. D33, 1616 (1986).

[22] B. Allen, Phys. Rev. D32, 3136 (1985).

[23] B. Allen and A. Folacci, Phys. Rev. D35, 3771 (1987).

[24] C. Pathinayake, A. Vilenkin, and B. Allen, Phys. Rev. D37, 2872 (1988).

[25] A. J. Tolley and N. Turok. Quantization of the massless minimally coupled scalar field and the $d S / C F T$ correspondence. hep-th/0108119.

[26] K. Kirsten and J. Garriga, Phys. Rev. D48, 567 (1993).

[27] S. W. Hawking and G. F. R. Ellis. The large scale structure of space-time. Cambridge University Press (1974).

[28] R. M. Wald. General relativity. The University of Chicago Press, USA (1984).

[29] R. Lehoucq, J.-P. Uzan, and J. Weeks, Kodai Math. J. 26, 119 (2003).

[30] E. T. Whittaker and G. N. Watson. A course of modern analysis. Cambridge University Press; 4th edition (1924).

[31] R. M. Wald. Quantum field theory in curved spacetime and black hole thermodynamics. The University of Chicago Press, USA (1994).

[32] N. M. J. Woodhouse. Geometric quantization. Oxford University Press, USA; 2nd edition (1997).

[33] E. Prugovečki. Quantum mechanics in Hilbert space. Academic Press, New York and London (1971).

[34] J. F. Barbero G., D. G. Vergel, and E. J. S. Villaseñor, in preparation. 